\title{
Pulmonary Artery Wall Thickness Assessed by Optical Coherence Tomography Correlates With Pulmonary Hemodynamics in Children With Congenital Heart Disease
}

\author{
Yukako Homma, MD; Yasunobu Hayabuchi, MD, PhD; \\ Akemi Ono, MD; Shoji Kagami, MD, PhD
}

\begin{abstract}
Background: Pulmonary arterial (PA) wall thickening evaluated by optical coherence tomography (OCT) has been reported in adults with PA hypertension. The purpose of this study was to evaluate the feasibility of OCT for preoperative assessment of the PA wall in children with congenital heart disease (CHD).

Methods and Results: Participants comprised 39 patients with ventricular septal defect, atrial septal defect, or patent ductus arteriosus. Attempts were made to evaluate vessels of various diameters using OCT. Clearly observed vessels that were optimal for evaluation were selected and classified into 4 subgroups by diameter of the lumen. Optimal depiction was obtained in 80 of 156 vessels in total, and $25(64.1 \%), 34(87.1 \%), 17(43.6 \%)$, and 4 vessels $(10.3 \%)$ in each of the $1.0-<2.0 \mathrm{~mm}, 2.0-<3.0 \mathrm{~mm}, 3.0-$ $<4.0 \mathrm{~mm}$, and $4.0-5.0 \mathrm{~mm}$ subgroups, respectively. Arterial walls in the $2.0-<3.0 \mathrm{~mm}$ subgroup were the most frequently delineated, and wall thickness correlated significantly with mean PA pressure, pulmonary vascular resistance index, pulmonary-to-systemic flow ratio, and PA capacitance index ( $r=0.56,0.52,0.37$, and -0.49 , respectively). The 3-layered appearance was delineated in 29 of 80 vessels $(36.2 \%)$. This feature had no significant correlation with pulmonary hemodynamics.
\end{abstract}

Conclusions: OCT represents a promising tool for evaluating the PA wall in children with CHD.

Key Words: Children; Congenital heart disease; Optical coherence tomography; Pulmonary arterial hypertension

$\mathbf{O}$ ptical coherence tomography (OCT) is an intravascular imaging modality with high resolution of up to $10-20 \mu \mathrm{m}$, which is around an order of magnitude greater than that achieved by intravascular ultrasonography (IVUS). OCT has thus contributed to clarification of the pathophysiology of coronary arteriosclerosis in adults, providing an effective tool for improving diagnosis and treatment. ${ }^{1,2}$

Previous pathological studies have shown pulmonary arterial (PA) wall thickening in adult PA hypertension (PAH) ${ }^{3,4}$ Recent advances in OCT studies have successfully revealed the same finding of PA wall thickening in adult PAH patients seen in pathological examination compared with controls., ${ }^{45}$ However, no reports appear to have described the evaluation of PA wall thickening in children.

In patients with congenital heart disease (CHD) with a left-to-right shunt, the volume and pressure overload on the pulmonary vascular bed provoke pulmonary arteriopathy in early infancy, inducing irreversible pulmonary vascular changes such as severe concentric intimal fibrosis, plexiform lesions, and dilatation lesions. ${ }^{6}$ In children with CHD showing increased pulmonary flow, irreversible pulmonary vascular changes are reported to begin progressing from over 6 months old. However, detailed images and changes over time before and after surgery are lacking, ${ }^{7}$ and OCT could conceivably aid in evaluating the course of pulmonary vascular changes in children with CHD.

The aim of this study was to investigate whether OCT represents a feasible tool for evaluating pathophysiological changes in the PA wall caused by pulmonary hemodynamic abnormalities in children with CHD.

Methods
Patients' Characteristics
We prospectively studied 39 infants and children ( 22 boys,
17 girls) with CHD showing increased pulmonary flow
because of a left-to-right shunt. Patients referred to the
Department of Pediatrics between May 2014 and October
2016 were included in the study. Anatomic diagnoses
were ventricular septal defect (VSD, $n=27)$, atrial septal
defect (ASD, $n=4)$, patent ductus arteriosus (PDA, $n=3$ ),
VSD with PDA (n=1), VSD with ASD and PDA ( $\mathrm{n}=1)$,
VSD with coarctation of the aorta ( $\mathrm{n}=2$ ) or interrupted
aortic arch $(\mathrm{n}=1)$ followed by arch reconstruction and PA
banding. Each diagnosis was made by echocardiography,

Received March 28, 2018; revised manuscript received May 23, 2018; accepted May 29, 2018; released online July 10 , 2018 Time for primary review: 27 days

Department of Pediatrics, School of Medicine, University of Tokushima, Tokushima, Japan

Mailing address: Yukako Homma, MD, Department of Pediatrics, School of Medicine, University of Tokushima, 3-18-15 Kuramoto-cho, Tokushima 770-8503, Japan. E-mail: farfalle31@hotmail.co.jp

ISSN-1346-9843 All rights are reserved to the Japanese Circulation Society. For permissions, please e-mail: cj@j-circ.or.jp 
A

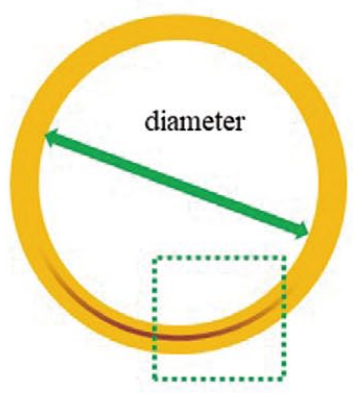

$\mathrm{C}$

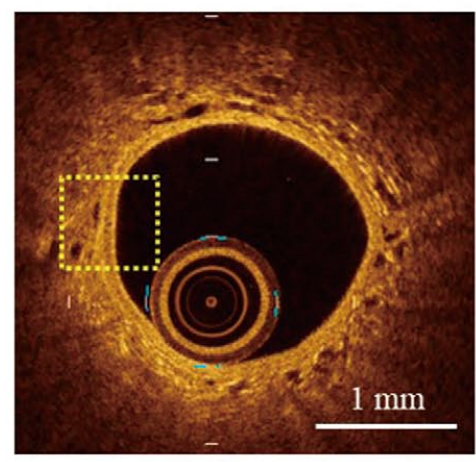

E

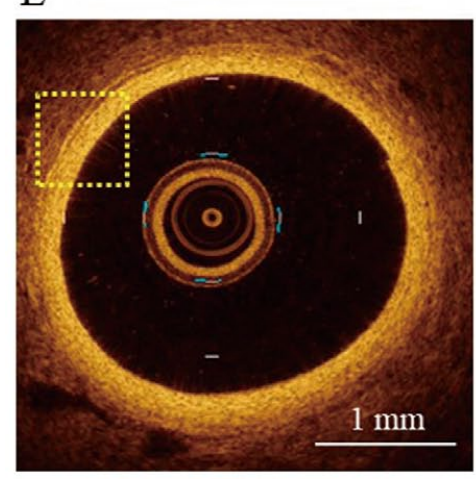

B

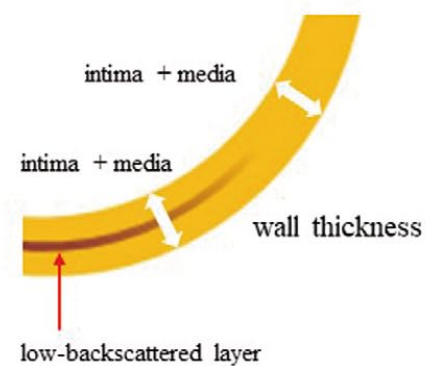

D

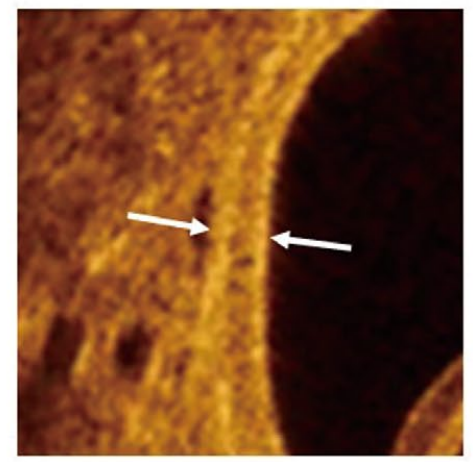

$\mathrm{F}$

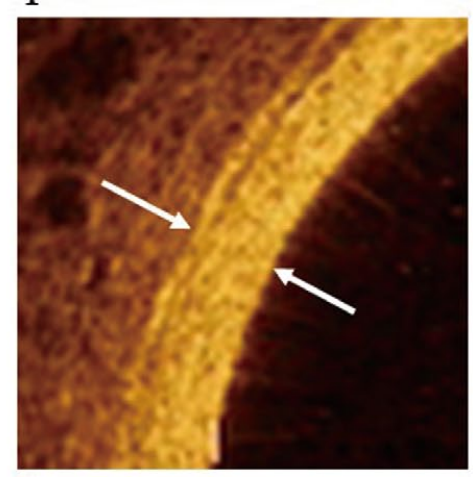

Figure 1. Images from optical coherence tomography (OCT) of the pulmonary arteries. (A) Vessel diameter was measured as shown by the green arrow. (B) Enlarged view depicting arterial wall thickness measured (shown by 2 white arrows), which indicates both intima-media layers. The intima-media layer was partially delineated as 3 high-lowhigh backscatter layers in some vessels, and continued smoothly into a single high-backscatter layer. (C-F) Vessel with high pulmonary arterial capacitance (pulmonary arterial capacitance index: PACi $5.4 \mathrm{~mL} / \mathrm{m}^{2} \cdot \mathrm{mmHg}$ ) shows a relatively thin arterial wall $(\mathbf{C}, \mathbf{D})$, compared with a vessel with low PAC (PACi $\left.2.1 \mathrm{~mL} / \mathrm{m}^{2} \cdot \mathrm{mmHg}\right)(\mathbf{E}, \mathbf{F})$. Arterial wall in $(\mathbf{C})$ shows delineation of 3 layers in the left side of the vessel, whereas the other segment of the wall has a single layer. multidetector-row computed tomography, and cardiac catheterization. Mean patient age was $2.0 \pm 2.0$ years (range, 2 months to 6 years). All protocols were approved by the Institutional Review Board of Tokushima University Hospital and conformed to the ethical guidelines of the 1975 Declaration of Helsinki. Written informed consent was given by the parents of all individual participants included in the study.

\section{Cardiac Catheterization}

All patients had undergone scheduled catheterization studies. Catheterization and angiography (Integris Allura 9 Biplane; Phillips Medical Systems, Best, the Netherlands) were performed using 4- to 6Fr catheters. All patients were intubated and examined under general anesthesia, with all data collected while breathing room air. Medications that could influence hemodynamics were not used during the procedures. No patients displayed complications of conventional angiography.
Right heart catheterization is part of the routine procedure for these patients in this institution, performed in the biplane anteroposterior and lateral positions. Catheters were connected to fluid-filled transducers and zeroed at atmospheric pressure. Pulmonary blood flow was calculated using the Fick principle. Pulmonary stroke volume was calculated as pulmonary blood flow per minute indexed to body surface area (BSA) divided by heart rate (HR). Pulmonary vascular resistance indexed to BSA (PVRi) was calculated using the standard formula: [mean PA pressure (mPAP)-PA wedge pressure] divided by pulmonary blood flow indexed to BSA (PVRi; expressed in Wood units $/ \mathrm{m}^{2}$ ). PA capacitance index (PACi) was calculated as [stroke volume indexed to $\mathrm{BSA}$ ] divided by PA pulse pressure (PACi; expressed in $\mathrm{mL} / \mathrm{m}^{2} \cdot \mathrm{mmHg}$ ).

\section{OCT Image Measurements}

OCT was performed for the purposes of this study after hemodynamic measurements. OCT images were acquired 


\begin{tabular}{|c|c|c|c|c|c|c|}
\hline & \multirow{3}{*}{$\begin{array}{c}\begin{array}{c}\text { Characteristics } \\
\text { of all patients }\end{array} \\
n=39\end{array}$} & \multicolumn{4}{|c|}{ Observed vessels sorted into subgroups by PA diameter } & \multirow{3}{*}{$P$ value } \\
\hline & & $1.0-<2.0 \mathrm{~mm}$ & $2.0-<3.0 \mathrm{~mm}$ & $3.0-<4.0 \mathrm{~mm}$ & $4.0-5.0 \mathrm{~mm}$ & \\
\hline & & $\mathrm{n}=25(64.1 \%)$ & $\mathrm{n}=34(87.1 \%)$ & $\mathrm{n}=17(43.6 \%)$ & $n=4(10.3 \%)$ & \\
\hline $\operatorname{Sex}(M / F)$ & $22 / 17$ & $18 / 7$ & $22 / 12$ & $11 / 6$ & $1 / 3$ & 0.35 \\
\hline Age (years) & $2.0 \pm 2.0$ & $2.0 \pm 2.0$ & $2.0 \pm 1.9$ & $1.9 \pm 2.1$ & $1.9 \pm 2.6$ & 0.98 \\
\hline Weight (kg) & $9.7 \pm 5.0$ & $10.0 \pm 5.2$ & $9.7 \pm 4.9$ & $9.8 \pm 5.6$ & $9.6 \pm 5.2$ & 0.99 \\
\hline Height $(\mathrm{cm})$ & $77.8 \pm 17.9$ & $78.0 \pm 17.1$ & $78.3 \pm 17.6$ & $77.5 \pm 18.6$ & $78.3 \pm 20.5$ & 0.99 \\
\hline $\operatorname{BSA}\left(m^{2}\right)$ & $0.4 \pm 0.2$ & $0.5 \pm 0.2$ & $0.5 \pm 0.2$ & $0.5 \pm 0.2$ & $0.4 \pm 0.2$ & 0.99 \\
\hline HR (beats/min) & $120.4 \pm 22.2$ & $116.8 \pm 23.3$ & $121.2 \pm 21.5$ & $129.3 \pm 23.6$ & $122.5 \pm 34.3$ & 0.42 \\
\hline $\mathrm{SBP}(\mathrm{mmHg})$ & $95.3 \pm 10.0$ & $97.6 \pm 9.9$ & $94.5 \pm 10.2$ & $95.4 \pm 10.2$ & $104.8 \pm 13.3$ & 0.35 \\
\hline $\mathrm{DBP}(\mathrm{mmHg})$ & $52.8 \pm 10.0$ & $54.1 \pm 10.6$ & $52.3 \pm 10.4$ & $50.9 \pm 10.1$ & $55.0 \pm 11.6$ & 0.70 \\
\hline Qp/Qs & $2.0 \pm 0.8$ & $1.8 \pm 0.8$ & $2.0 \pm 0.8$ & $2.0 \pm 0.8$ & $2.3 \pm 0.9$ & 0.44 \\
\hline RVSP (mmHg) & $39.0 \pm 15.6$ & $41.3 \pm 16.5$ & $38.6 \pm 15.4$ & $37.5 \pm 14.8$ & $32.0 \pm 13.5$ & 0.69 \\
\hline RVEDP (mmHg) & $7.7 \pm 2.8$ & $8.0 \pm 2.0$ & $7.7 \pm 2.9$ & $7.4 \pm 2.7$ & $9.3 \pm 2.9$ & 0.42 \\
\hline SPAP (mmHg) & $32.4 \pm 13.5$ & $33.5 \pm 14.4$ & $31.1 \pm 12.6$ & $31.8 \pm 13.0$ & $27.0 \pm 2.6$ & 0.95 \\
\hline DPAP (mmHg) & $12.2 \pm 5.2$ & $12.5 \pm 5.8$ & $12.1 \pm 4.5$ & $12.7 \pm 4.8$ & $12.8 \pm 1.9$ & 0.67 \\
\hline mPAP $(\mathrm{mmHg})$ & $21.6 \pm 8.2$ & $22.1 \pm 8.7$ & $21.1 \pm 8.0$ & $21.5 \pm 7.8$ & $19.5 \pm 2.4$ & 0.98 \\
\hline PAPP (mmHg) & $20.2 \pm 10.6$ & $21.0 \pm 11.4$ & $19.0 \pm 9.5$ & $19.0 \pm 10.1$ & $14.3 \pm 2.1$ & 0.78 \\
\hline PVRi (Wood Units/m²) & $1.6 \pm 0.8$ & $1.6 \pm 0.8$ & $1.5 \pm 0.8$ & $1.4 \pm 0.8$ & $1.2 \pm 0.4$ & 0.77 \\
\hline $\mathrm{PACi}\left(\mathrm{mL} / \mathrm{m}^{2} \cdot \mathrm{mmHg}\right)$ & $3.2 \pm 1.6$ & $2.8 \pm 1.1$ & $3.3 \pm 1.6$ & $3.1 \pm 1.4$ & $3.8 \pm 1.5$ & 0.52 \\
\hline PA wall thickness (mm) & $0.19 \pm 0.06$ & $0.10 \pm 0.05$ & $0.19 \pm 0.05$ & $0.23 \pm 0.05^{\star}$ & $0.24 \pm 0.09$ & 0.004 \\
\hline 3-layered delineation & $29 / 80(36.2 \%)$ & $11(44.0 \%)$ & $13(38.2 \%)$ & $4(23.5 \%)$ & $1(25.0 \%)$ & 0.55 \\
\hline
\end{tabular}

Data are given as mean $\pm S D$. ${ }^{*} P=0.001$ vs. $1.0-<2.0 \mathrm{~mm}$ subgroup, and $\mathrm{P}=0.04$ vs. $2.0-<3.0 \mathrm{~mm}$ subgroup. $B S A$, body surface area; $D B P$, diastolic blood pressure; DPAP, diastolic pulmonary arterial pressure; HR, heart rate; mPAP, mean pulmonary arterial pressure; PA, pulmonary artery; PACi, pulmonary arterial capacitance indexed to body surface area; PAPP, pulmonary arterial pulse pressure; PVRi, pulmonary vascular resistance indexed to body surface area; $\mathrm{Qp} / \mathrm{Qs}$, pulmonary-to-systemic blood flow ratio; RVEDP, right ventricular end-diastolic pressure; RVSP, right ventricular systolic pressure; SPAP, systolic pulmonary arterial pressure.

using a commercially available ILUMEN FD-OCT Imaging System (St. Jude Medical, St. Paul, MN, USA). Images of 2 segmental PAs of the right or left inferior lobe were obtained in both the still state and during pullback at a rate of $54 \mathrm{~mm} / \mathrm{s}$. Observation of as many vessels with different diameters as possible was performed. A low-molecularweight dextran flush technique was used to clear away blood and obtain high-quality images. No patient suffered complications of OCT imaging.

The vessel diameter and wall thickness were evaluated with image analysis software (Image J 1.45; National Institutes of Health, Bethesda, MD, USA). The diameter of the vessel lumen was measured between opposing luminal outlines of the intima (Figure 1A). Diameter was scaled in the diastolic phase to avoid overestimation, because vessels dilate in the systolic phase. Arterial wall thickness was measured between the luminal outline of the intima and the outer line of the media (measured as the single intima-media layer). The intima-media layer was partially delineated as 3-layered high-low-high backscatter in some vessels. We considered that the middle low-backscatter layer would be the border of the intima and media, or the luminal layer of media, because the 3-layered appearance continued smoothly into a single high-backscatter layer (Figure 1B). In vessels with a 3-layered appearance, we measured these layers as the wall thickness accounting for the intima-media layers.

\section{Statistical Analysis}

Categorical variables are expressed as absolute values and percentages, and continuous variables as mean \pm standard deviation (SD). Measurements of PA wall thickness were repeated 3 times, and average values were used for the analysis. Those who measured the wall thickness were blinded to the hemodynamics of the patients. Correlations between PA wall thickness and mPAP, systolic PA pressure (SPAP), PVRi, pulmonary-to-systemic blood flow ratio (Qp/Qs), PACi, age, body weight, BSA, and systolic blood pressure were examined using Pearson's correlation coefficient. Mean demographic and baseline hemodynamic values for each subgroup were compared by the KruskalWallis test followed by the Dunnett test, as appropriate. Values of $\mathrm{P}<0.05$ were considered statistically significant. Commercially available statistical software (JMP for Windows version 12.1.0; SAS, Cary, NC, USA) was used for all analyses. Intraclass correlation coefficients (ICCs) were calculated to assess intra- and interobserver agreement in measurements of PA wall thickness. Intra- and interobserver reproducibilities of measurements were assessed by Bland-Altman analysis. Interobserver variability was assessed by analyzing data recorded from 20 randomly selected patients at 8 -week intervals by 2 separate observers, blinded to each other's results. For intraobserver variability, data were analyzed twice by the same observer, at an interval of 8 weeks.

\section{Results}

\section{Data Acquisition}

Because the diameter of the distal end of the OCT catheter is $1 \mathrm{~mm}$, we could not observe vessels with a diameter less than $1 \mathrm{~mm}$. Also, we could not clearly depict images of 

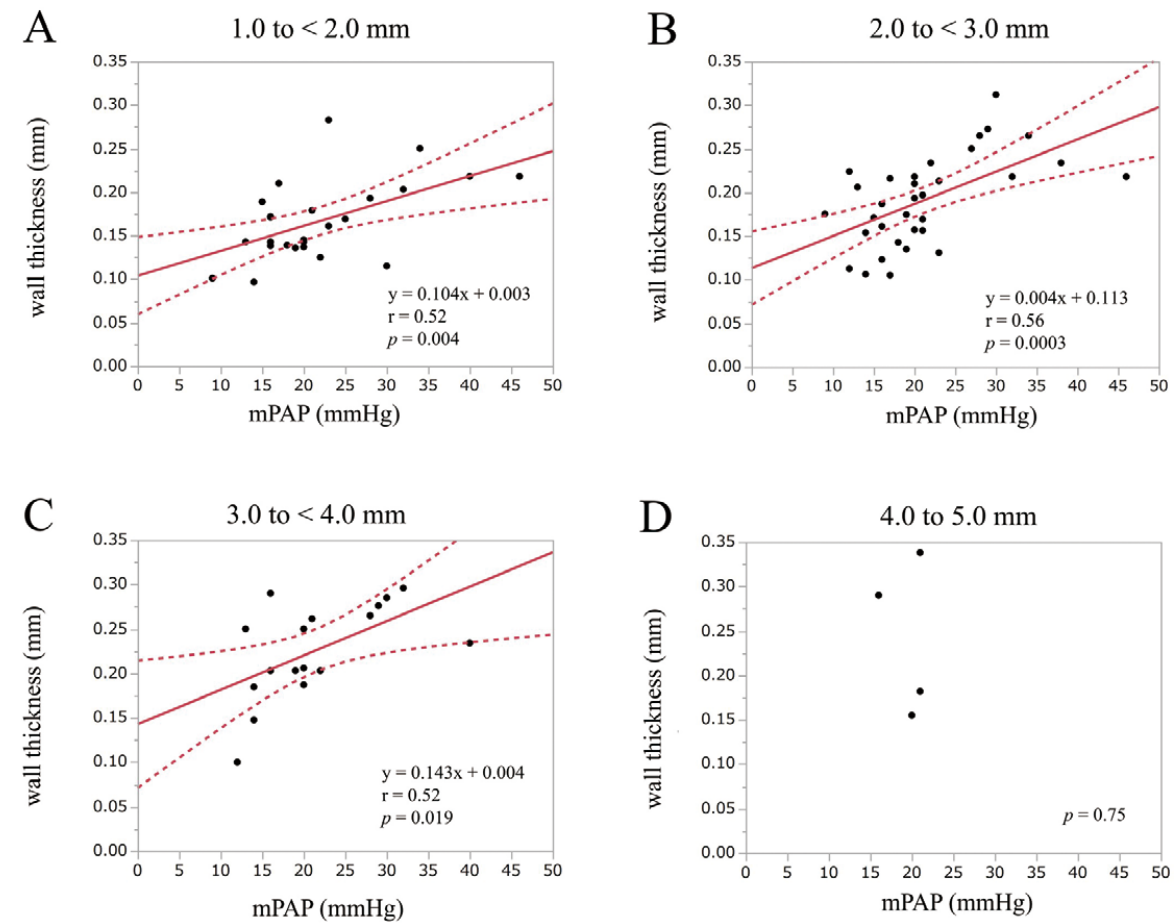

Figure 2. Relationships between pulmonary arterial wall thickness and mean pulmonary arterial pressure (mPAP) in each subgroup. Linear regression lines with 95\% confidence intervals are indicated by dotted lines. Wall thickness showed a significant positive correlation with mPAP in vessel subgroups with a diameter of $1.0-<2.0 \mathrm{~mm}, 2.0-<3.0 \mathrm{~mm}$, and $3.0-<4.0 \mathrm{~mm}$.

vessels with a diameter over $5 \mathrm{~mm}$ because of the ineffective dextran flush. For these reasons, clearly observed lumen diameters ranged from 1.31 to $5.0 \mathrm{~mm}$. Attempts were made to evaluate vessels of various diameters using OCT in all patients. Observable vessel diameters were classified into 4 subgroups in intervals of $1.0 \mathrm{~mm}$ from $1.0 \mathrm{~mm}$ to $5.0 \mathrm{~mm}$. Therefore, we evaluated 156 vessels in total, which consisted of 4 subgroups per individual, in the 39 patients. The $2.0-<3.0 \mathrm{~mm}$ subgroup was the most clearly observed among the 39 cases $(1.0-<2.0 \mathrm{~mm}$ : 25 cases, $64.1 \%$; 2.0 $<3.0 \mathrm{~mm}$ : 34 cases, $87.1 \%$; 3.0-<4.0 mm: 17 cases, $43.6 \%$; $4.0-5.0 \mathrm{~mm}$ : 4 cases, $10.3 \%$ ). Although we made efforts to obtain suitable images of diameters at all levels of the PA, $35.9 \%, 12.9 \%, 56.4 \%$, and $89.7 \%$ of vessels were not clearly depicted in the $1.0-<2.0 \mathrm{~mm}, 2.0-<3.0 \mathrm{~mm}, 3.0-<4.0 \mathrm{~mm}$, and $4.0-5.0 \mathrm{~mm}$ subgroups, respectively. These images were excluded from the subsequent investigation. In the end, 80 PA walls were clearly observed by OCT within all patients, and were used for analysis in this study.

\section{Clinical Characteristics of Patients and Each Vessel Subgroup}

Clinical characteristics of observable PA walls with demographic and baseline hemodynamic data sorted by diameter are shown in Table. Of the 80 vessels observed in this study, $52(65 \%)$ were in male patients, and $28(35 \%)$ were in female patients. No significant differences in demographic and baseline hemodynamic data were seen between the 4 subgroups. In contrast, PA wall thickness was significantly different between the subgroups. Larger vessels had thicker walls. The PA wall in the $3.0-<4.0 \mathrm{~mm}$ subgroup was significantly thicker than in the $1.0-<2.0 \mathrm{~mm}$ and $2.0-<3.0 \mathrm{~mm}$ subgroups $(\mathrm{P}=0.001$ and 0.04 , respectively). We performed the subsequent evaluation regarding the relationship between PA wall thickness and pulmonary hemodynamics in each subgroup sorted by vessel diameter, which was considered to be reasonable on the basis of these results.

\section{OCT Images and Pulmonary Hemodynamics}

Representative OCT images of the PA walls are shown in patients with low PAP and high PACi (Figure 1C,D) and high PAP and low PACi (Figure 1E,F). Relationships between the PA wall and mPAP, SPAP, PVRi, Qp/Qs, and $\mathrm{PACi}$ in each diameter subgroup are shown in Figures 2-6. Wall thickness showed significant positive correlations with mPAP (Figure 2), SPAP (Figure 3), and PVRi (Figure 4) in vessels with a diameter of $1.0-<4.0 \mathrm{~mm}$. A significant positive correlation was also seen between Qp/Qs and PA wall thickness in vessels with a diameter of $1.0-<3.0 \mathrm{~mm}$ (Figure 5). PACi showed a significant negative correlation with wall thickness in vessels with a diameter of 2.0 $<4.0 \mathrm{~mm}$ (Figure 6).

Significant differences in wall thickness were also shown between the PAH group (mPAP $\geq 25 \mathrm{mmHg}$ ) and non-PAH group (mPAP $\leq 20 \mathrm{mmHg}$ ) in the $2.0-<3.0 \mathrm{~mm}$ (Figure 7A) and $3.0-<4.0 \mathrm{~mm}$ (Figure 7B) diameter subgroups (median [interquartile range], 0.17 [0.15-0.19] vs. 0.25 [0.23-0.28], $\mathrm{P}<0.0001$; and 0.20 [0.17-0.23] vs. 0.27 [0.23-0.32], $\mathrm{P}=0.032$, respectively). 

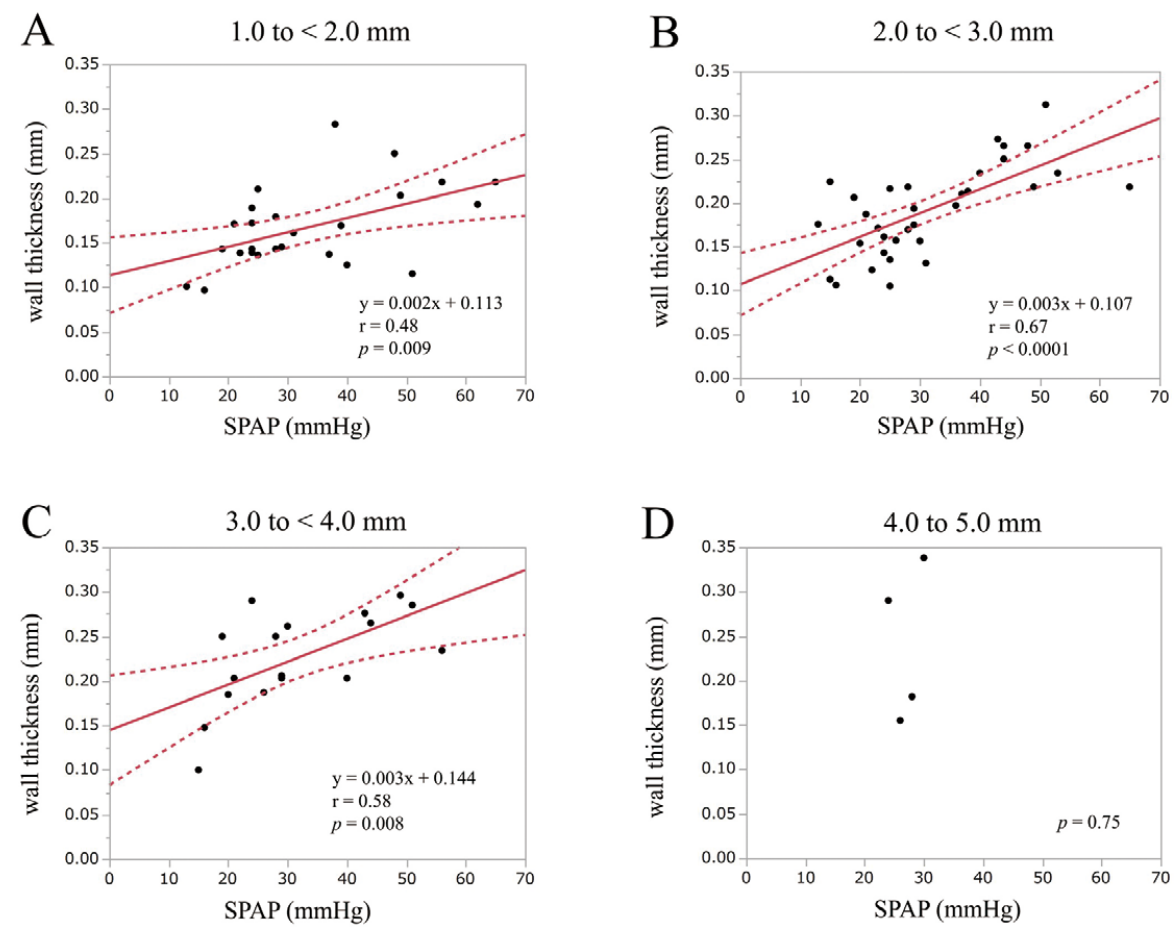

Figure 3. Relationships between pulmonary arterial wall thickness and systolic pulmonary arterial pressure (SPAP) in each diameter subgroup. Linear regression lines with 95\% confidence intervals are indicated by dotted lines. Wall thickness showed a significant positive correlation with SPAP in vessels with a diameter of $1.0-<2.0 \mathrm{~mm}, 2.0-<3.0 \mathrm{~mm}$, and $3.0-<4.0 \mathrm{~mm}$.
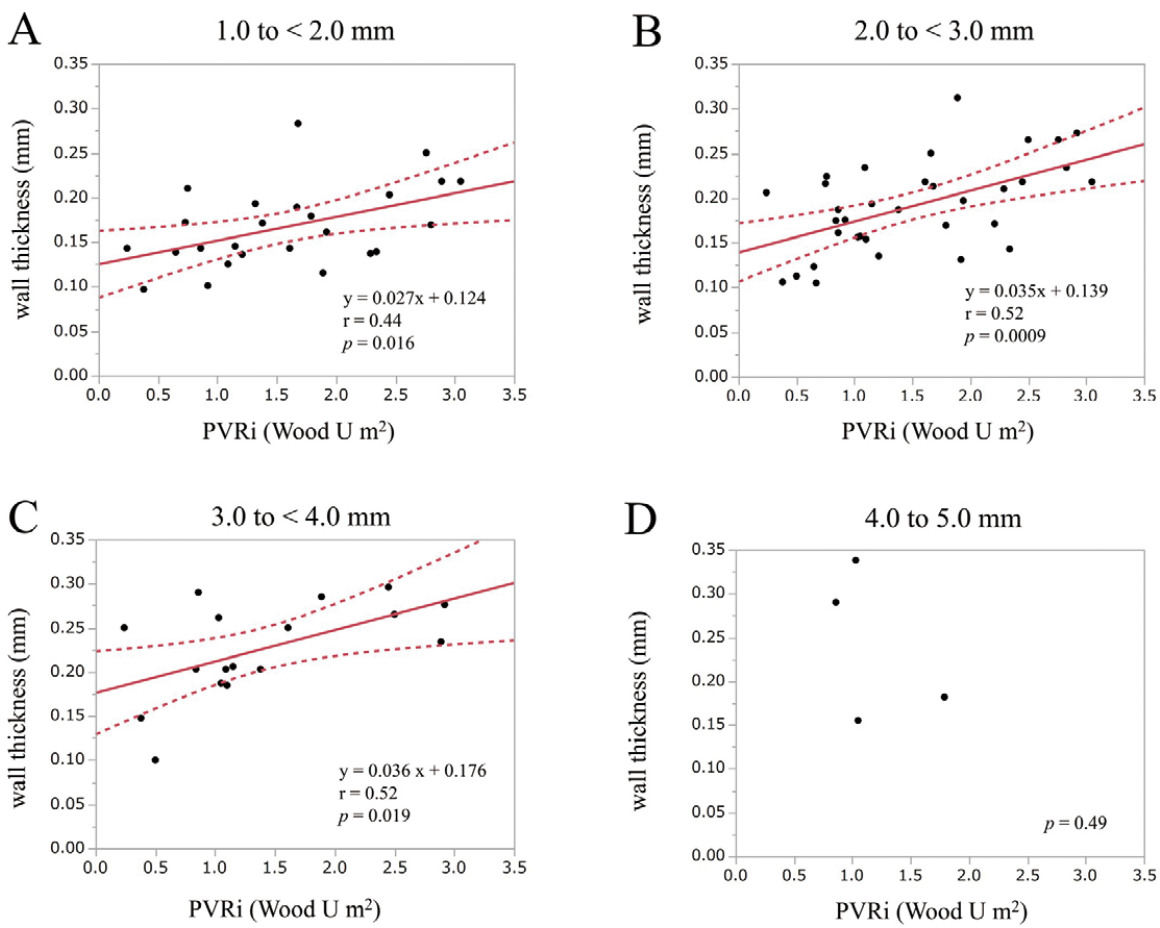

Figure 4. Relationships between pulmonary arterial wall thickness and pulmonary vascular resistance indexed to body surface area (PVRi) in each diameter subgroup. Linear regression lines with 95\% confidence intervals are indicated by dotted lines. Wall thickness showed significant positive correlation with PVRi in vessels with a diameter of $1.0-<2.0 \mathrm{~mm}, 2.0-<3.0 \mathrm{~mm}$, and $3.0-$ $<4.0 \mathrm{~mm}$. 

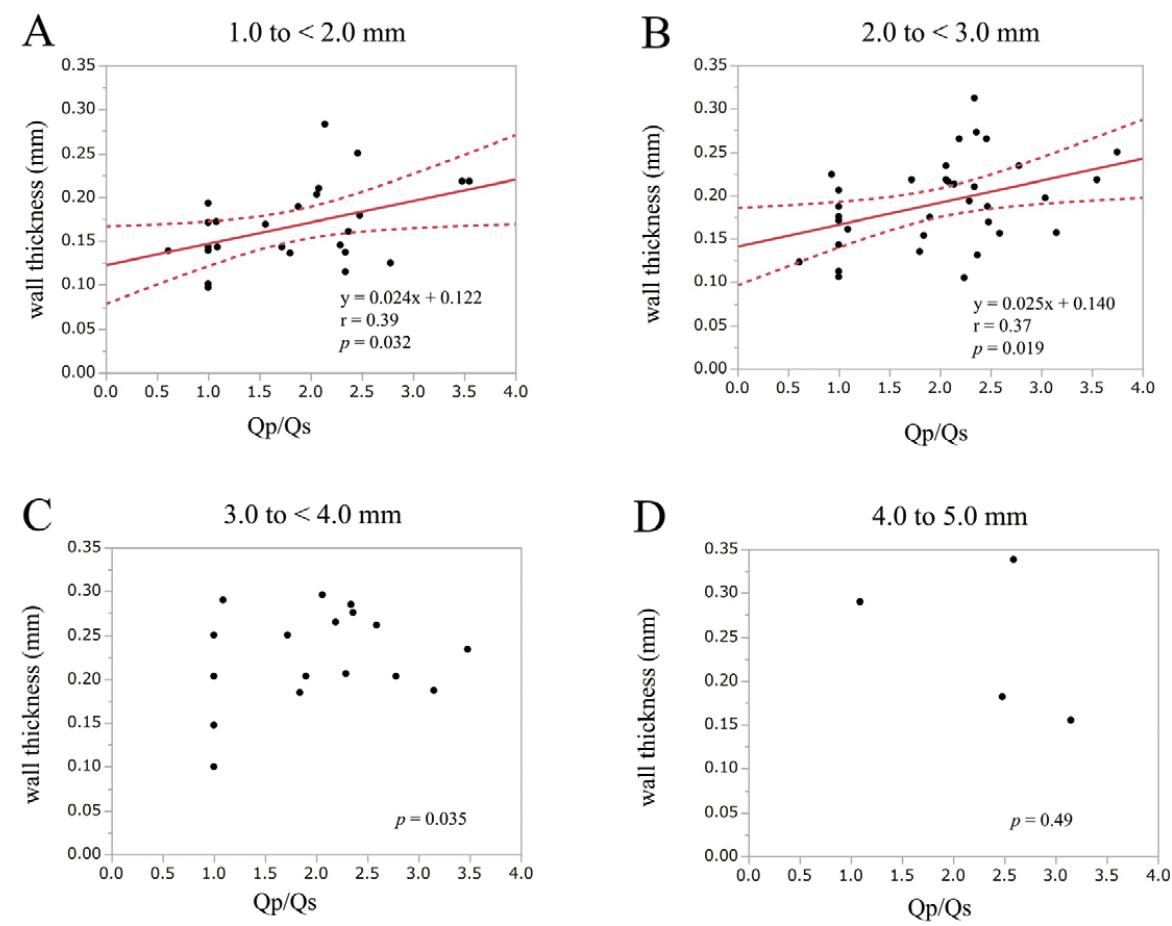

Figure 5. Relationships between pulmonary arterial wall thickness and pulmonary-to-systemic blood flow ratio (Qp/Qs) in each diameter subgroup. Linear regression lines with 95\% confidence intervals are indicated by dotted lines. A significant positive correlation was also seen for $\mathrm{Qp} / \mathrm{Qs}$ in vessels with a diameter of $1.0-<2.0 \mathrm{~mm}$ and $2.0-<3.0 \mathrm{~mm}$.
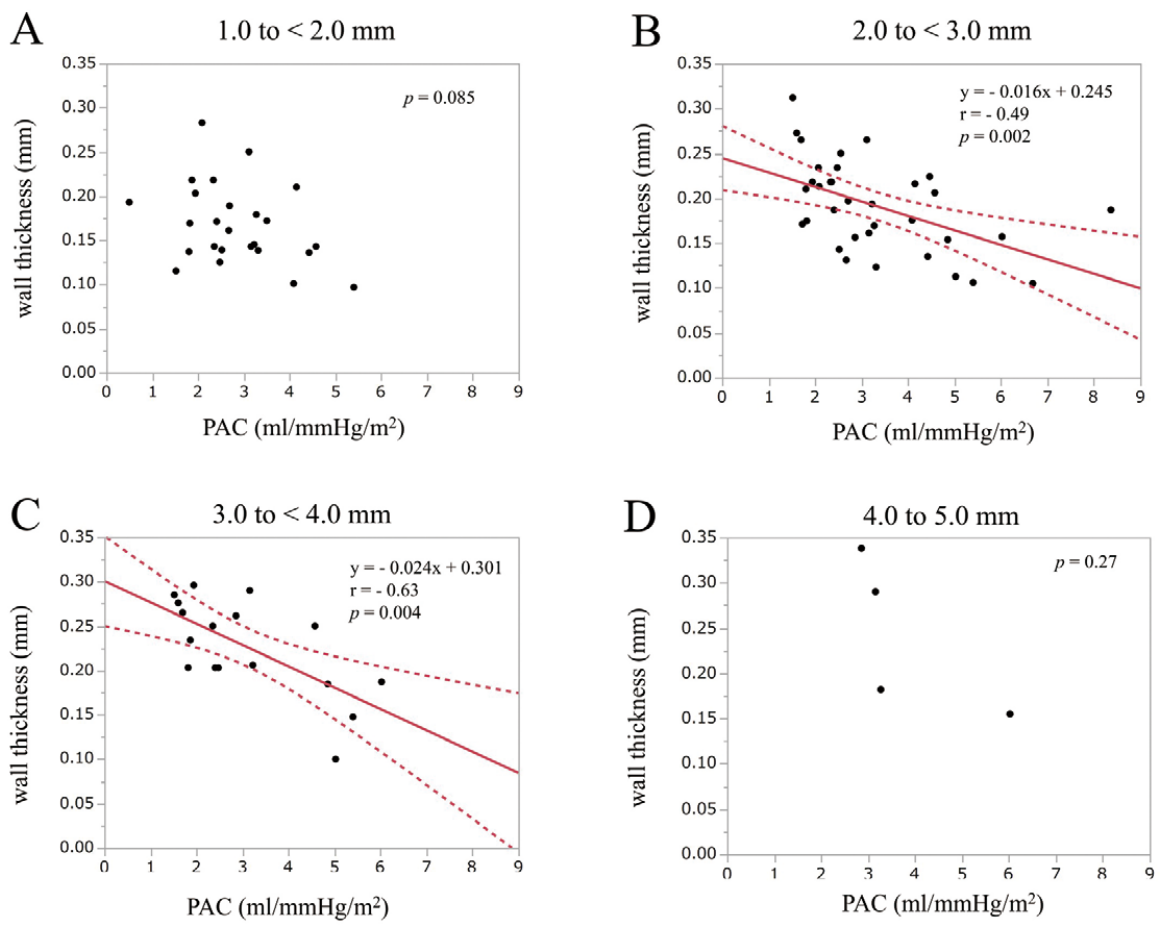

Figure 6. Relationships between pulmonary arterial wall thickness and pulmonary arterial capacitance index (PACi) in each diameter subgroup. Linear regression lines with 95\% confidence intervals are indicated by dotted lines. PACi showed a significant negative correlation with wall thickness in vessels with a diameter of $2.0-<3.0 \mathrm{~mm}$ and $3.0-<4.0 \mathrm{~mm}$. 


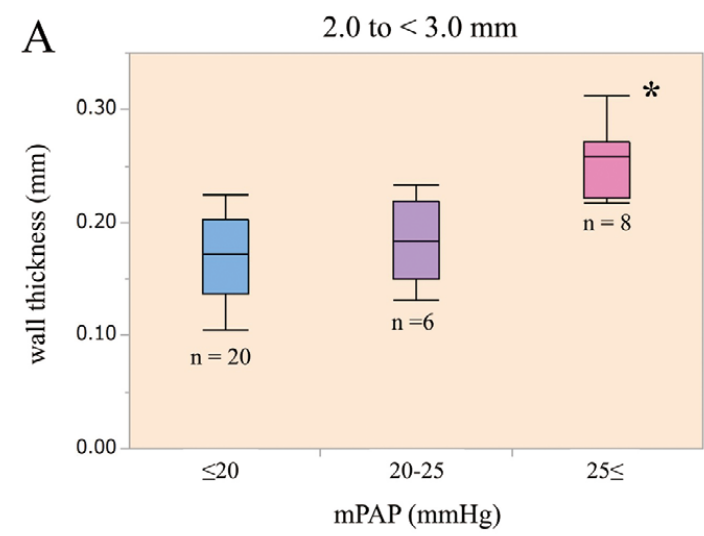

B

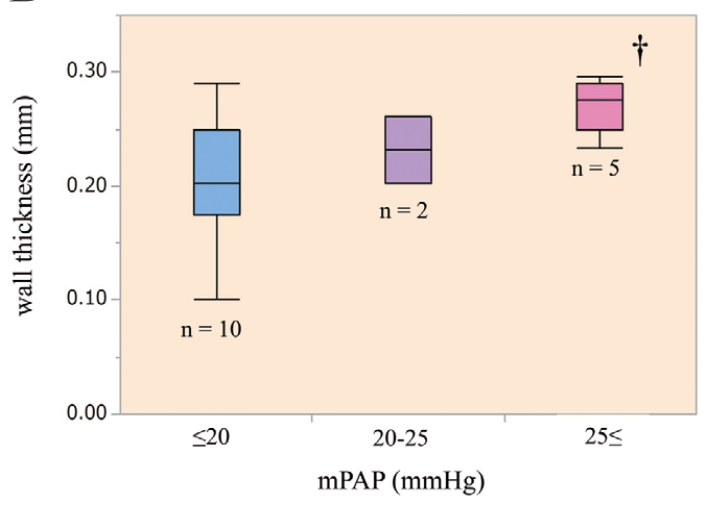

Figure 7. Pulmonary arterial wall thickness compared for the presence, borderline, and absence of pulmonary hypertension in $2.0-<3.0 \mathrm{~mm}(\mathbf{A})$ and $3.0-<4.0 \mathrm{~mm}$ (B) diameter subgroups. Boxes, interquartile range; Central line, median; Whiskers, minimum and maximum. ${ }^{\star} \mathrm{P}<0.0001$ vs. $\leq 20 \mathrm{mmHg}$ group and $\mathrm{P}=0.007$ vs. $20-25 \mathrm{mmHg}$ group. ${ }^{\dagger} \mathrm{P}=0.032$ vs. $\leq 20 \mathrm{mmHg}$ group.

\section{Three-Layered Structure of PA Wall}

The 3-layered appearance was delineated in 29 of 80 vessels $(36.2 \%)$. No significant differences in SPAP, mPAP, PACi, Qp/Qs, and PVRi were apparent between patients with and without delineation of the 3 layers $(30.3 \pm 12.0$ vs. $32.7 \pm 13.4 \mathrm{mmHg}, \mathrm{P}=0.39 ; 20.6 \pm 6.6$ vs. $21.9 \pm 8.6 \mathrm{mmHg}$, $\mathrm{P}=0.46 ; 3.2 \pm 1.4$ vs. $3.1 \pm 1.4 \mathrm{~mL} / \mathrm{m}^{2} \cdot \mathrm{mmHg}, \mathrm{P}=0.75 ; 1.8 \pm 0.7$ vs. $2.1 \pm 0.8, \mathrm{P}=0.09$; and $1.4 \pm 0.8$ vs. $1.5 \pm 0.8$ Wood units $/ \mathrm{m}^{2}$, $\mathrm{P}=0.68$; respectively).

\section{Reproducibility}

In the measurements of PA wall thickness, the ICC for interobserver reproducibility was 0.96 (95\% confidence interval (CI), 0.95-0.97). The ICC for intraobserver reproducibility was $0.83(95 \% \mathrm{CI}, 0.81-0.85)$. Inter- and intraobserver reproducibility obtained from Bland-Altman plots (bias $\pm 1.96 \mathrm{SD}$; 95\% limit of agreement) were $0.001 \pm 0.012 \mathrm{~mm}$ and $0.037 \pm 0.023 \mathrm{~mm}$, respectively.

\section{Discussion}

The present investigation demonstrated that OCT can depict the PA walls in children, and showed significant correlations between PA wall thickness and pulmonary hemodynamics. This is the first study to assess the PA wall appearance in children with CHD.

In our study, the PA walls were relatively most easily delineated in vessels with a diameter of $2.0-<3.0 \mathrm{~mm}$, regardless of differences in age, body size, or hemodynamics. This was probably because of our simple procedural approach to catheterization and the moderate vessel diameter allowing effective flush of the dextran.

Most early vascular lesions of PAH are found in pulmonary arterioles with diameters ranging from 300 to $500 \mu \mathrm{m}$, although as the disease advances, larger-diameter arteries also show medial thickening and microstructural alterations. ${ }^{8-10}$ Significant results in our study were more frequently shown in larger-diameter vessels than in arterioles, which were originally considered as the site of PAH lesions. The vessels observed in this study could conceivably reflect the hemodynamics induced by the pathology of arteriolar lesions, or might themselves actually be primary lesions of PAH. The pathologic features of PA wall thickening observed by OCT in adult PAH patients are reported as including neointimal formation, intimal fibrosis, and medial thickening. ${ }^{2,3}$ However, there are not sufficient studies comparing OCT findings and histopathology. Further studies are required to elucidate these issues in CHD children.

\section{Correlation Between PA Wall Thickness and Pulmonary Hemodynamics}

We identified a significant correlation between PA wall thickness and PAP using OCT in children with CHD. On histopathological examination, PAH is reportedly associated with medial/intimal hypertrophy. ${ }^{11,12}$ Lau et al reported diffuse thickness of the PA walls in vessels in the range of $2-5 \mathrm{~mm}$ diameter using IVUS in adult PAH patients, ${ }^{10}$ and our results using OCT in children resemble those findings. The results in our study suggested the potential of OCT in CHD for describing subtle lesions in the very early stages of PAH and identifying the development of PA remodeling as previously reported in adults. ${ }^{13}$ OCT may be effective in assessing response to $\mathrm{PAH}$ therapy, and the extent to which pulmonary pressure is reversible during treatment.

PA wall thickness showed significant correlations with PACi and PVRi. Domingo et al found that the severity of OCT-measured 'intimal fibrosis' showed a more significant negative correlation with PACi than with PVRi. ${ }^{14}$ Reports have also described wall fibrosis identified on OCT as predictive of unfavorable clinical outcomes during mid-term follow-up. ${ }^{15}$ Further investigation of the utility of OCT in evaluating PACi and PVRi may shed some light on the management of PAH.

\section{Architecture of the PA Wall}

OCT images of the PA wall are known to show a single layer with homogeneous, signal-rich bands. ${ }^{2}$ In this study, we observed 3 layers in some PA walls and these were considered to represent the intima-media layer, because the 3-layered appearance continued smoothly into the single intima-media layer. The exact timing of obtaining images, the location of the wire, the distance between the wire and the PA wall, and the degree of blood wash-out are considered as factors that could influence the delineation of the 3 layers. PAP and PAC did not show significant relationships with the delineation of 3 layers in this study. Li et al reported that the PA media is difficult to distinguish from the intima and adventitia on OCT, ${ }^{\mathbf{1 6}}$ but the arteries 
observed in that report were cadaveric tissues, so it seems likely that there will be differences in the image characteristics between cadaveric and living arteries. The 3-layered appearance may reflect pathological changes or may be an effect of the catheter procedure in OCT. The implications of a 3-layered architecture and comparisons with pathological observations of the PA wall need to be investigated.

\section{Study Limitations}

Several limitations must be considered with regard to this study. First, because OCT imaging needs a blood-free imaging field achieved by transient occlusion of the proximal flow or by the injection of dextran, some difficulty is encountered in clearly visualizing relatively wider vessels. ${ }^{2,15}$ Unlike the situation in adults, balloon occlusion is difficult in children, because the vessels are not large enough for insertion of larger sheaths. A dextran flush may change the PAP and PA diameter, and we cannot rule out the possibility of some influence of dextran on PA wall thickness. Second, the depth of penetration for OCT is about $2 \mathrm{~mm}$, half that of IVUS, which may result in limited visualization of walls in severely thickened vessels. ${ }^{16}$ Third, in this study, the PA walls were most frequently observed in the $2.0-<3.0 \mathrm{~mm}$ diameter subgroup, because of the relatively simple approach to obtaining stable images during the catheter procedure. In addition, these vessels were considered to have a moderate diameter allowing effective dextran flush within the range of OCT performance. We need to collect more data on other diameter vessels and lobes to evaluate differences in the pathological changes to the PA walls. Determining the correspondence between sites measured by OCT and examined histologically represents another task. ${ }^{14}$ Fourth, children without heart diseases were not included in this investigation to ensure normal PA features and measurements were obtained. However, ethical difficulties exist regarding the performance of catheterization or OCT observation in children with normal hearts. We consider that children without high PAP may offer an alternative to normal healthy children. Besides, further investigation regarding the pathological differences in various diseases or the postoperative changes are desirable in the future. Finally, the OCT image wire is fragile and expensive, and the costs and benefits of PA OCT have yet to be clarified. ${ }^{15}$

\section{Conclusions}

This represents the first study to establish a population of preoperative CHD children for observation of the PA walls using OCT. Observation of the PA walls in children with CHD is as yet poorly documented. The results demonstrated that PA wall thickness correlated significantly with pulmonary hemodynamic parameters. Eisenmenger syndrome, cyanotic heart disease, or children after Fontan operation might show different findings from typical PAH as shown in this study. OCT offers a promising new tool to evaluate the process of PA lesions in children with CHD with increased pulmonary flow.

\section{Funding \\ No funding was received for this study. \\ Conflicts of Interest}

The authors declare that they have no conflicts of interest.

\section{References}

1. Tearney GJ, Regar E, Akasaka T, Adriaenssens T, Barlis P, Bezerrea HG, et al. Consensus standards for acquisition, measurement, and reporting of intravascular optical coherence tomography studies. JACC 2012; 59: 1058-1072.

2. Jorge E, Baptista R, Calisto J, Faria H, Monteiro P, Pan M, et al. Optical coherence tomography of the pulmonary arteries: A systematic review. Int J Cardiol 2016; 67: 6-14.

3. Brunner NW, Zamanian RT, Ikeno F, Mitsutake Y, Connolly AJ, Shuffle E, et al. Optical coherence tomography of pulmonary arterial wall in humans and pigs (Sus scrofa domesticsus). Comp Med 2015; 65: 217-224.

4. Pietra GG, Capron F, Stewart S, Leone O, Humbert M, Robbins IM, et al. Pathologic assessment of vasculopathies in pulmonary hypertension. J Am Coll Cardiol 2004; 16: 25-32.

5. Regar E, Liqthart J, Bruining N, van Soest G. The diagnostic value of intracoronary optical coherence tomography. Herz 2011; 36: $417-429$.

6. Yamaki S, Wagenvoort CA. Comparison of primary plexogenic arteriopathy in adults and children: A morphometric study in 40 patients. Br Heart J 1985; 54: 428-434.

7. Masaki N, Saiki Y, Endo M, Maeda K, Adachi O, Akiyama M, et al. Evidence of pulmonary vascular reverse remodeling after pulmonary artery banding performed in early infancy in patients with congenital heart defects. Circ J 2018; 82: 684-690.

8. Heath D, Helmholz HF, Burchell HB, DuShane JW, Edwards JE. Graded pulmonary vascular changes and hemodynamic findings in cases of atrial and ventricular septal defect and patent ductus arteriosus. Circulation 1958; 18: $1155-1166$.

9. Prapa M, McCarthy KP, Dimopoulos K, Sheppard MN, Krexi D, Swan L, et al. Histopathology of the great vessels in patients with pulmonary arterial hypertension in association with congenital heart disease: Large pulmonary arteries matter too. Int J Cardiol 2013; 168: 2248-2254.

10. Lau EM, Iyer N, Ilsar R, Bailey BP, Adams MR, Celermajer DS Abnormal pulmonary artery stiffness in pulmonary arterial hypertension: In vivo study with intravascular ultrasound. $P L O S$ One 2012; 7: e33331.

11. Palevsky HI, Schloo BL, Pietra GG, Weber KT, Janicki JS, Rubin E, et al. Primary pulmonary hypertension: Vascular structure, morphometry, and responsiveness to vasodilator agents. Circulation 1989; 80: 1207-1221.

12. Zaiman A, Fijalkowska I, Hassoun PM, Tuder RM. One hundred years of research in the pathogenesis of pulmonary hypertension. Am J Respir Cell Mol Biol 2005; 33: 425-431.

13. Dai Z, Fukumoto Y, Tatebe S, Sugimura K, Miura Y, Nochioka $\mathrm{K}$, et al. OCT imaging for the management of pulmonary hypertension. JACC Cardiovasc Imaging 2014; 7: 843-845.

14. Domingo E, Grinqnola JC, Aquilar R, Montero MA, Arrendondo C, Vázquez M, et al. In vivo assessment of pulmonary arterial wall fibrosis by intravascular optical coherence tomography in pulmonary arterial hypertension: A new prognostic marker of adverse clinical follow-up. Open Respir Med J 2013; 5: $26-32$.

15. Hong C, Wanq W, Zhong NS, Zeng GQ, Wu H. Using optical coherence tomography to detect peripheral pulmonary thrombi. Clin Med J 2012; 125: 3171-3174.

16. Li N, Zhang S, Hou J, Jang IK, Yu B. Assessment of pulmonary artery morphology by optical coherence tomography. Heart Lung Circ 2012; 21: 778-781. 